\title{
Water-retaining properties of NCZ composite dust suppressant and its wetting ability to hydrophobic coal dust
}

\author{
Jianguo Liu ${ }^{1,2,3}$ (i) Shu Wang ${ }^{1,2,3} \cdot$ Longzhe Jin ${ }^{1,2,3} \cdot$ Tianyang Wang ${ }^{1} \cdot$ \\ Zihao Zhou ${ }^{1} \cdot$ Jingge $\mathrm{Xu}^{1}$
}

Received: 26 December 2019/Revised: 12 February 2020/Accepted: 27 November 2020/Published online: 5 January 2021

(C) The Author(s) 2021

\begin{abstract}
Coal dust is a primary threat to underground coal miners. The most common approach to control coal dust is hydraulic methods, such as water spray and coal seam water injection. To improve the dust suppressant efficiency of hydraulic methods, a novel chemical composite dust suppressant, called NCZ, was prepared in this study using calcium chloride $\left(\mathrm{CaCl}_{2}\right)$, magnesium chloride $\left(\mathrm{MgCl}_{2}\right)$, and nonionic surfactants using a thermal synthesis method. The waterretaining properties of $\mathrm{NCZ}$ powder and its solutions were characterized using the water absorption rate $(W A R)$ and evaporation rate $(E R)$, respectively, and the wetting abilities of the NCZ solutions on coal dust were tested using the initial contact angle (ICA) and sink rate $(S R)$. The results indicate that the NCZ solutions have anti-evaporation effects, and the $E R$ of the solution with a $20.0 \mathrm{wt} \% \mathrm{NCZ}$ is reduced by $11.7 \%$ compared with that of clean water. Furthermore, NCZ solutions have remarkable enhancement effects on the wettability of coal dust. The ICA and SR of clean water and the NCZ solution at $20.0 \mathrm{wt} \%$ are $141.9^{\circ}$ and $0 \mathrm{mg} / \mathrm{s}$, and $29.3^{\circ}$ and $1.46 \mathrm{mg} / \mathrm{s}$, respectively. Finally, quantitative relationships between the solution surface tension and the ICA and IR were established using the least squares method. This study provides a new product for dust suppression in underground mines, which is significant for the optimum applied concentration of dust suppressant in mining operations.
\end{abstract}

Keywords Coal dust $\cdot$ Hydrophobicity $\cdot$ Dust suppressant $\cdot$ Wettability $\cdot$ Water retention

Shu Wang

ustbwangshu@ustb.edu.cn

$\triangle$ Longzhe Jin

lzjin@ustb.edu.cn

1 School of Civil and Resource Engineering, University of Science and Technology Beijing, Beijing 100083, China

2 Key Laboratory of High-Efficient Mining and Safety of Metal Mines of the Ministry of Education, University of Science and Technology Beijing, Beijing 100083, China

3 Mine Emergency Technology Research Center, University of Science and Technology Beijing, Beijing 100083, China

\section{Introduction}

In recent decades, as the level of mining mechanization has continuously increased, coal dust concentration in underground mines has similarly grown, and the associated exposure time of high concentrations of coal dust to workers has increased (Xu et al. 2018; Shi et al. 2019). The increasing cases of pneumoconiosis (CWP) and coal dust explosion accidents in recent years have forced enterprises and governments worldwide to focus their attention on coal dust suppression (Hall et al. 2019). For instance, the permissible exposure limit (PEL) of respiratory coal dust in the US was reduced from 2.0 to $1.5 \mathrm{mg} / \mathrm{m}^{3}$ in 2016 (Xu et al. 2018). In China, the Air Pollutant Discharge Standard (GB16297-1996) was revised by China's Ministry of Environmental Protection in 2017, and the requirements for fugitive emission control measures in mining processes 
were enhanced (Zhou G et al. 2018; Zhou L et al. 2018; Zhou Q et al. 2018).

There has been considerable research to efficiently control coal dust in underground mines by studying its generation mechanism, diffusion process, and suppressant technologies. To date, the most common approach to control coal dust is hydraulic methods, such as water spray, coal seam water injection, and chemical dust suppression. In these cases, wetting fluids (mainly water or water solutions) are used to wet the coal dust particles, increase their mass, and facilitate their settling (Kollipara et al. 2014; Zhou et al. 2020; Jin et al. 2019; Liu et al. 2019a). Therefore, the wetting ability of fluids on coal dust is significant to achieve high dust suppressant efficiencies.

It is widely accepted that studying the influence of the physicochemical properties of dust on its wettability is important (Xu et al. 2017; Wang et al. 2017, 2019; Liu et al. 2019b). In addition, decreasing the surface tension of wetting fluids is another effective approach to improve the wettability of coal dust (Xu et al. 2018; Shi et al. 2019; Meng et al. 2019). Consequently, numerous studies have focused on developing chemical dust suppressants. Adding surfactants is the most common method to reduce the surface tension of water, and the effects of the concentration and species (including cationic, anionic, and nonionic) of surfactants on the solution surface tension have been intensely investigated (Meng et al. 2019; Shi et al. 2019; Xi et al. 2019). In addition, the synergistic effects of different surfactant species (Xi et al. 2017; Bao et al. 2020; Zhou et al. 2020), the compatibility of surfactants with various kinds of chlorine salts (e.g., $\mathrm{NaCl}, \mathrm{CaCl}_{2}$ ) (Jin et al. 1999; $\mathrm{Ni}$ et al. 2019), and surfactant-magnetized water (Zhou G et al. 2018; Zhou L et al. 2018; Zhou Q et al. 2018) have been investigated. Nevertheless, few chemical dust inhibitors have been widely applied in the field due to their low dust suppression efficiency, high cost, and other factors (Xu et al. 2018). Therefore, developing a chemical coal dust suppressant that has good dust inhibition efficiency and is convenient for field applications is required to control coal dust in underground mines.

In this study, a novel composite chemical suppressant, called NCZ, was prepared using calcium chloride $\left(\mathrm{CaCl}_{2}\right)$, magnesium chloride $\left(\mathrm{MgCl}_{2}\right)$, and nonionic surfactants using a thermal synthesis method for the hydrophobicity of coal dust. The water-staining properties of NCZ powder and its solutions were then characterized using water absorption and anti-evaporation tests, respectively. Next, the wettability of the NCZ solutions with different concentrations on coal dust were evaluated by measuring the sink rate $(S R)$ and initial contact angles (ICA) on a coal dust platelet. Finally, the effects of the solution surface tension on the wettability of coal dust were discussed, and their quantitative relationship was fit using the least squares method.

\section{Materials and experiments}

\subsection{Coal dust sample}

The experimental coal dust was collected using a hang plate from a return airway in Yongcheng coalfield in Henan Province and sieved with a 200 mesh $(\leq 74 \mu \mathrm{m})$ in a laboratory. The proximate and ultimate analyses of the coal dust were conducted following the GB/T 212-2008 and GB/T 31391-2015 standards, respectively. The results are listed in Table 1. The dynamic contact angles between the coal dust and deionized water were measured at $7.5 \mathrm{~Hz}$ for $10 \mathrm{~s}$ using a Theta Lite TL101 apparatus (Finland). As shown in Fig. 1, the contact angles at 0 and $10 \mathrm{~s}$ were $141.9^{\circ}$ and $109.6^{\circ}$, respectively. Therefore, the coal dust is almost considered a super-hydrophobic material (contact angle $\left.>150^{\circ}\right)($ Notsu et al. 2005).

\subsection{Preparation of NCZ dust suppressant}

A thermal synthesis method was utilized to prepare the NCZ dust suppressant to ensure all the components were fully mixed. As shown in Fig. 2, $100 \mathrm{~g}$ of a $\mathrm{CaCl}_{2}$ and $\mathrm{MgCl}_{2}$ mixture (4:1 mass ratio; both materials have a relatively large water-absorbing capacity (Jin et al. 1999; Ni et al. 2019) and can prevent settled dust from flying again) was dissolved in water. The solution was heated, and when the temperature reached $120-140{ }^{\circ} \mathrm{C}, 5 \mathrm{~g}$ of a non-ionic surfactant mixture, i.e., primary alcohol ethoxylate (JFC) and Triton $\mathrm{X}-100$ at a 1:1 mass ratio, was added to the solution. The solution was then poured into a stainless-steel mold, which was cooled in a water bath to room temperature before being unloaded. The NCZ infiltration sticks were obtained, which were placed directly into water injection holes on a coal seam to enhance the wettability of the coal body.

Table 1 Proximate and ultimate analyses of the experimental coal dust

\begin{tabular}{|c|c|c|c|c|c|c|c|c|}
\hline \multicolumn{4}{|c|}{ Proximate analysis (wt $\%)$} & \multicolumn{5}{|c|}{ Ultimate analysis (wt\%, daf) } \\
\hline$M_{\mathrm{ad}}$ & $A_{\text {ad }}$ & $V_{\text {daf }}$ & $\mathrm{FC}_{\mathrm{ad}}$ & $\mathrm{C}$ & $\mathrm{H}$ & $\mathrm{N}$ & $\mathrm{S}$ & $\mathrm{O}^{*}$ \\
\hline 0.98 & 39.25 & 10.72 & 49.05 & 51.33 & 2.31 & 0.67 & 0.48 & 45.21 \\
\hline
\end{tabular}


Finally, the NCZ infiltration sticks were pulverized to form a mesh powder $(\leq 1.70 \mathrm{~mm})$ to test its enhancement effect on the wettability of coal dust. The NCZ infiltration sticks before (a) and after (b) pulverizing are shown in Fig. 3.

\subsection{Surface tension test}

The pendant drop method (Berry et al. 2015) was used to measure the dynamic surface tension of clean water and the NCZ solutions at $7.5 \mathrm{~Hz}$ over $30 \mathrm{~s}$ using a Theta Lite TL101 apparatus (Fig. 4). The average values were used for further calculations.

\subsection{Water-retaining test}

The water-retaining ability of the dust suppressant significantly affects its dust suppression efficiency because it can decrease the probability of settled dust flying again through absorption and anti-evaporating water (Zhang et al. 2018).

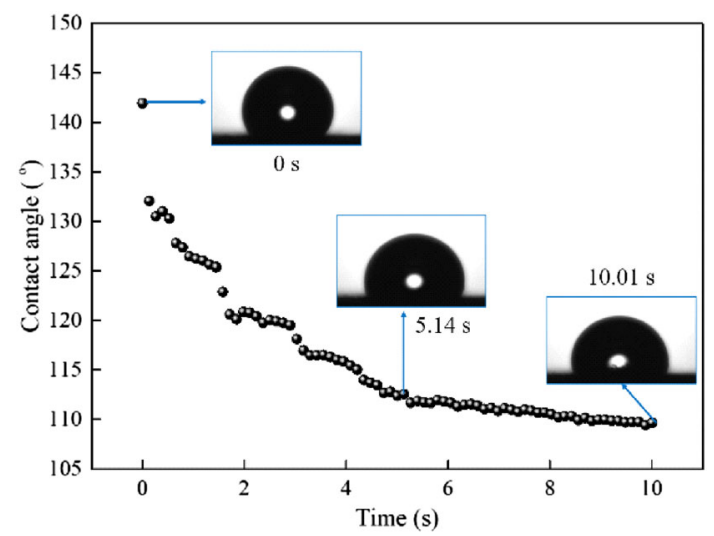

Fig. 1 Dynamic contact angles of the experimental coal dust on deionized water within $10 \mathrm{~s}$

\subsubsection{Water-absorption test}

Approximately $1 \mathrm{~g}$ of the NCZ powder (Fig. 3b) was placed in a culture dish $(\varphi=58 \mathrm{~mm})$ and kept in a laboratory environment at $17 \pm 1{ }^{\circ} \mathrm{C}$ and $(25 \pm 5) \%$ humidity
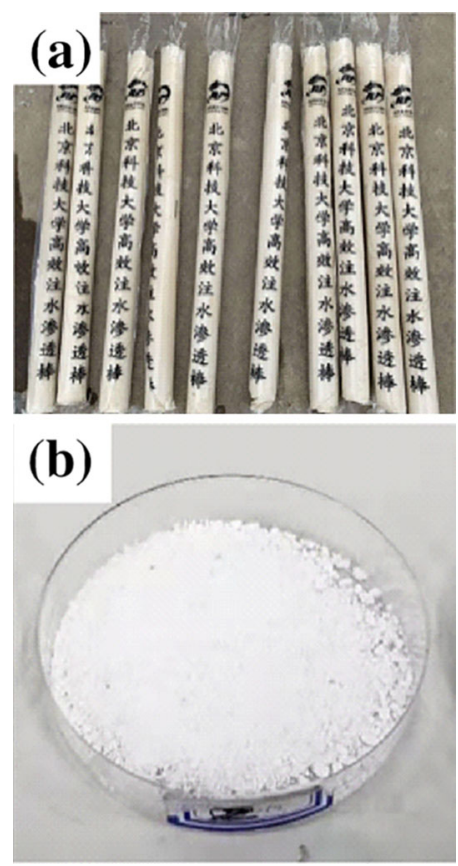

Fig. 3 Photographs of NCZ infiltration sticks before (a) and after (b) pulverizing

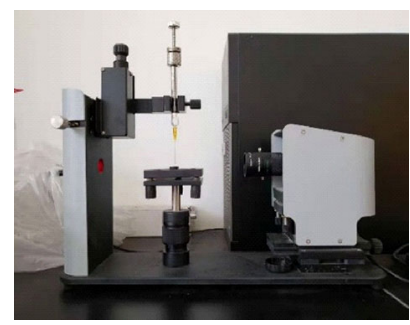

Fig. 4 Photographs of the Theta Lite TL101 apparatus

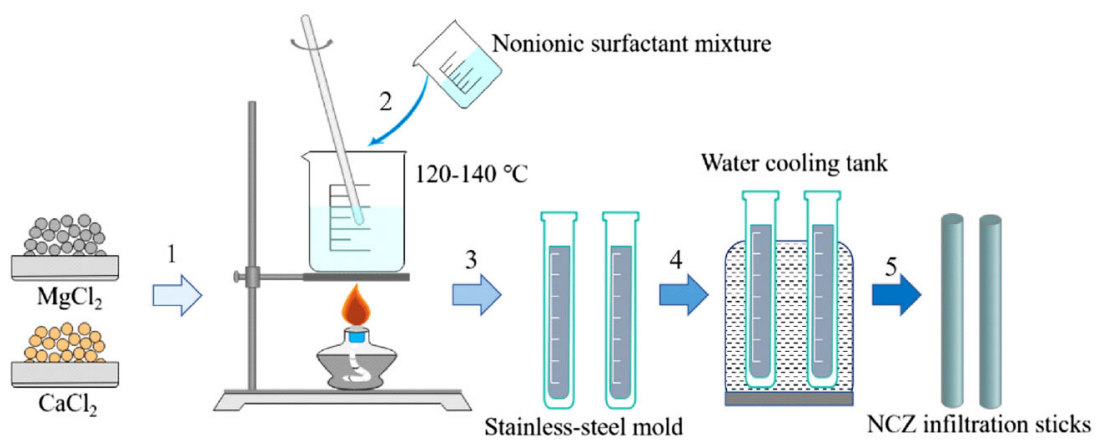

Fig. 2 Preparation process of the NCZ infiltration sticks 
for $26 \mathrm{~h}$. Next, the powder mass was examined every $2 \mathrm{~h}$ using an analytical balance (sensitivity of $0.0001 \mathrm{~g}$ ), and the water absorption rate (WAR) was calculated using Eq. (1).

$W A R=\frac{m_{t}-m_{0}}{m_{0} t}$,

where, $m_{0}$ and $m_{t}$ are the masses of the NCZ powder at time 0 (initial mass) and $t$, respectively.

\subsubsection{Anti-evaporation test}

Approximately $20 \mathrm{~mL}$ of NCZ solutions with concentrations of $0 \%$ (clean water), $0.1 \%, 0.5 \%, 1.0 \%, 5.0 \%, 10.0 \%$, and $20.0 \%$ were poured into culture dishes $(\varphi=80 \mathrm{~mm})$ and placed in a laboratory environment (the same conditions used for the water-absorption test) for $24 \mathrm{~h}$. Their masses were measured and recorded every $2 \mathrm{~h}$, and the evaporation rate $(E R)$ was calculated using Eq. (2).

$E R=\frac{m_{0}-m_{t}}{t}$

The averages were considered for further calculations.

\subsection{Wettability test}

(1) Contact angle test

The dynamic contact angles between the coal dust and NCZ solutions at various concentrations were measured per the discussion in Sect. 2.1, and the ICA value was selected for further analysis ( $\mathrm{Li}$ et al. 2013).

(2) Sink test

Compared with other laboratory static tests, the sink test is the most reliable method to quantify the wettability of coal dust (Chen et al. 2019). In this study, $0.1-0.5 \mathrm{~g}$ of experimental coal dust was placed on the surface of the NCZ solutions, and the time (sink time) taken for all coal dust to disappear from the surface was recorded (Shi et al. 2019; Chen et al. 2019). The sink rate $(S R)$ is calculated using Eq. (3):

$S R=\frac{m}{t}$

where, $m$ is the mass of coal dust. The sink tests were repeated three times, and the average value was taken.

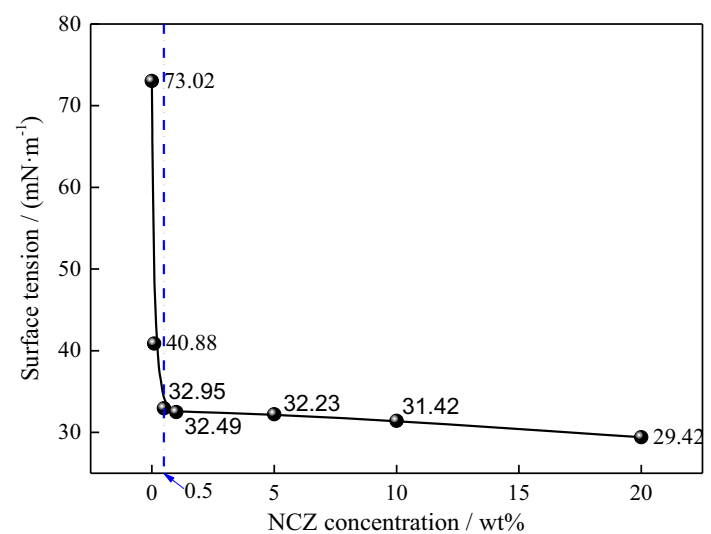

Fig. 5 Curve of the surface tension for the NCZ solution at different concentrations

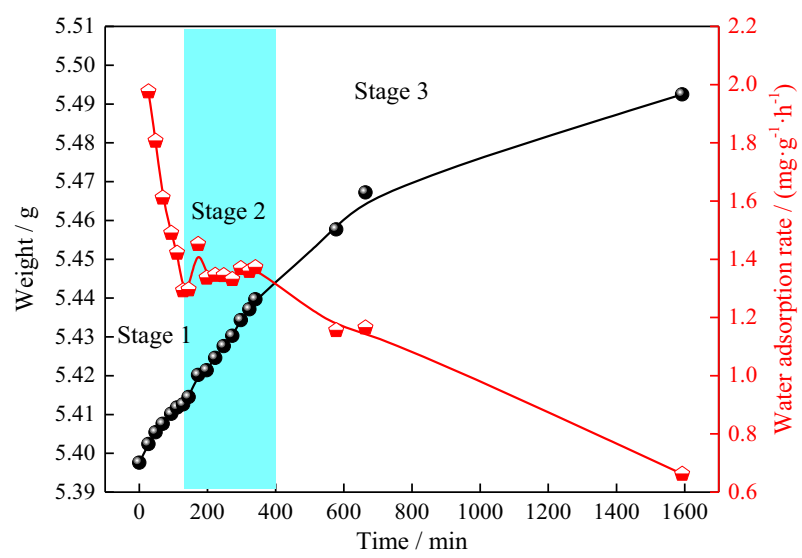

Fig. 6 Variations in the mass and WAR of NCZ powder with time

\section{Results and discussion}

\subsection{Changes in surface tension of NCZ solutions}

The variability of the surface tension for the NCZ solutions (0.0 wt $\%, \quad 0.1 \mathrm{wt} \%, \quad 0.5 \mathrm{wt} \%, \quad 1.0 \mathrm{wt} \%, \quad 5.0 \mathrm{wt} \%$, $10.0 \mathrm{wt} \%$, and $20.0 \mathrm{wt} \%$ ) is shown in Fig. 5. It is noted that the solution surface tension reduced sharply from 73.02 to $32.95 \mathrm{mN} / \mathrm{m}$ with the addition of $0.5 \mathrm{wt} \% \mathrm{NCZ}$. With further increases in the NCZ concentration, the solution surface tension reduced more slowly and reduced only by $3.53 \mathrm{mN} / \mathrm{m}$ as the $\mathrm{NCZ}$ concentration increased from $0.5 \mathrm{wt} \%$ to $20.0 \mathrm{wt} \%$. The critical micelle concentration (CMC) (Xu et al. 2018) of the NCZ solution is thus $0.5 \mathrm{wt} \%$. The surfactant molecules form a unimolecular layer at the CMC on the solution surface, which results in a reduced surface tension. Multiple layers of surfactant molecules are then formed on the surface when the concentration is $>\mathrm{CMC}$, but this has little effect on the solution surface tension (Xu et al. 2018). Therefore, the surface tension of the NCZ solution is reduced sharply at 
concentrations less than $0.5 \mathrm{wt} \%$, and when the $\mathrm{NCZ}$ concentration is greater than $0.5 \mathrm{wt} \%$, the solution surface tension is reduced at a relatively slow rate.

\subsection{Water-retaining property of NCZ powder and solutions}

\subsubsection{Water absorption process of NCZ powder}

Changes in the NCZ powder mass and its WAR with time are shown in Fig. 6, where three distinct stages are observed. In stage 1 , the physical adsorption of water dominated from $0-130 \mathrm{~min}$ as the NCZ powder is much drier than air, and the WAR decreased linearly from 1.98 to $1.29 \mathrm{mg} / \mathrm{g} \mathrm{h}$. The $W A R$ then began to fluctuate steadily between 1.3 and $1.4 \mathrm{mg} / \mathrm{g}$ h from 130-400 min (stage 2). Here, the chemical absorption dominates, which is attributed to the hygroscopic properties of $\mathrm{CaCl}_{2}$ and $\mathrm{MgCl}_{2}$. In stage 3 (> $400 \mathrm{~min}$ ), the mass of the NCZ powder increased slowly, and the water adsorption process stopped

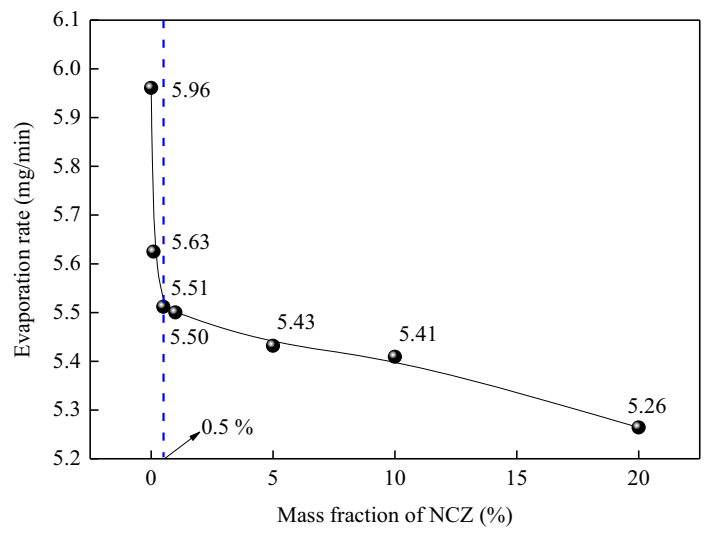

Fig. 7 Evaporation rate of $\mathrm{NCZ}$ solutions at different concentrations when the equilibrium point was reached. That is, the WAR of the NCZ powder was equal to the $E R$ of water. At the equilibrium point, the mass of the NCZ powder does not change, and the WAR becomes 0 .

\subsubsection{Evaporation properties of NCZ solutions}

The evaporation rate of the dust suppressant solution is directly related to the possibility of the settled dust flying again. Therefore, investigating the evaporation properties of the NCZ solution is important to understand its dust suppressed performance. It is widely accepted that the $E R$ of a solution increases as its surface tension decreases because the intermolecular forces inside the solution are reduced. The dust suppressant efficiency is improved by adding surfactant into water, but this accelerates the evaporation of moisture in coal dust and lets the settled dust become airborne. To induce anti-evaporation of the dust inhibitor solutions, stronger water-absorbent salts, i.e., $\mathrm{CaCl}_{2}$ and $\mathrm{MgCl}_{2}$, were added into the $\mathrm{NCZ}$ suppressant. Figure 7 shows the $E R$ curve of the NCZ solutions at differing concentrations. The $E R$ rapidly decreased first before slowly decreasing with an increasing $\mathrm{NCZ}$ concentration with a transition point of $0.5 \mathrm{wt} \%$. This is consistent with the trend of the surface tension (Fig. 5), which illustrates that the NCZ dust suppressant can not only effectively reduce the solution surface tension but can also enhance the anti-evaporation properties of the solutions. The $E R$ of the $20 \mathrm{wt} \%$ NCZ solution decreased by $11.7 \%$ compared with that of clean water.

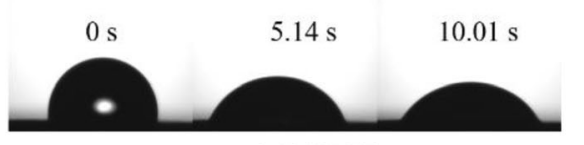

$0.1 \% \mathrm{NCZ}$
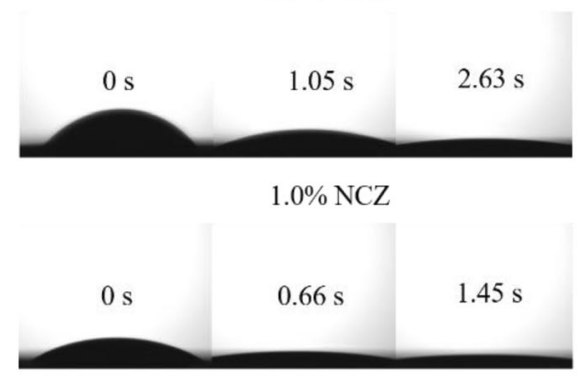

$10.0 \% \mathrm{NCZ}$
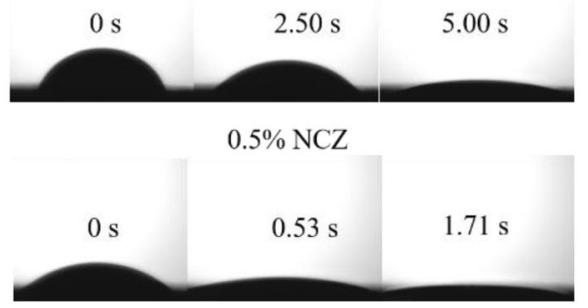

$5.0 \% \mathrm{NCZ}$

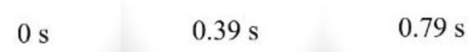

Fig. 8 Images of water solution drops on compressed coal dust pellets 


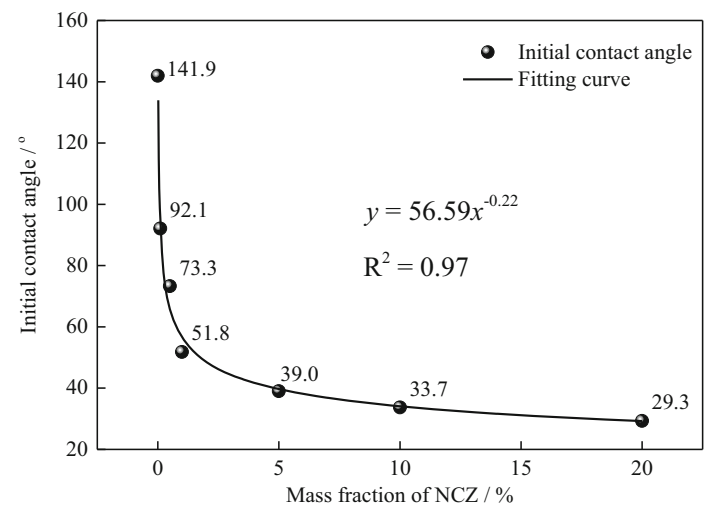

Fig. 9 Initial contact angle of NCZ solutions on the coal dust surface

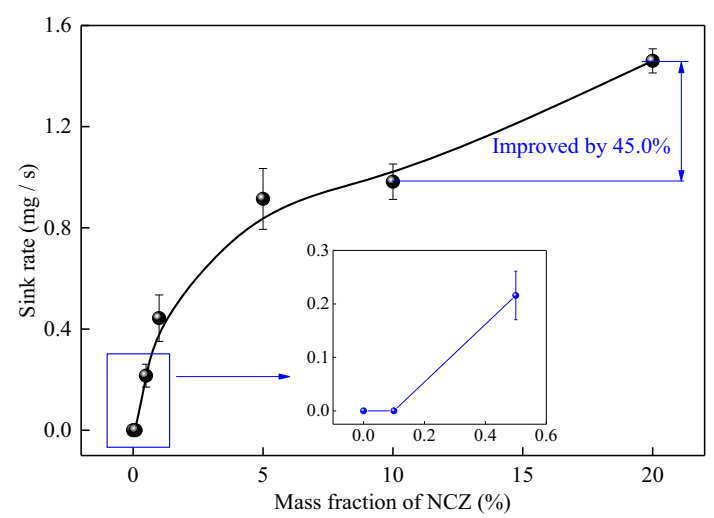

Fig. 10 Sink rate curve of coal dust in the NCZ solutions

\subsection{Enhancement in wettability of coal dust by NCZ solutions}

\subsubsection{Contact angle}

Figure 8 shows several images of contact angles at various times. As the ICA is an effective method to evaluate the wettability of coal dust ( $\mathrm{Li}$ et al. 2013), it was selected to evaluate the wettability of NCZ.

As shown in Fig. 9, the ICA decreased gradually for greater NCZ concentrations following a power function trend $\left(R^{2}=0.97\right)$. However, unlike changes in the surface tension, when the NCZ concentration was $>0.5 \mathrm{wt} \%$ (CMC), the ICA reduced significantly with concentration. That is, the ICA decreased by $54.0 \%$ when the NCZ concentration increased from $0.5 \mathrm{wt} \%$ to $10.0 \mathrm{wt} \%$. The optimal wetting concentration was $20 \mathrm{wt} \%$ with a minimum contact angle of $29.3^{\circ}$. This indicates that increasing the NCZ concentration beyond $0.5 \mathrm{wt} \%$ can significantly enhance the wettability of coal dust although this has little effect on the solution surface tension. Thus, the solution at the CMC point is far from its strongest wetting performance on coal dust. This is significant when selecting the
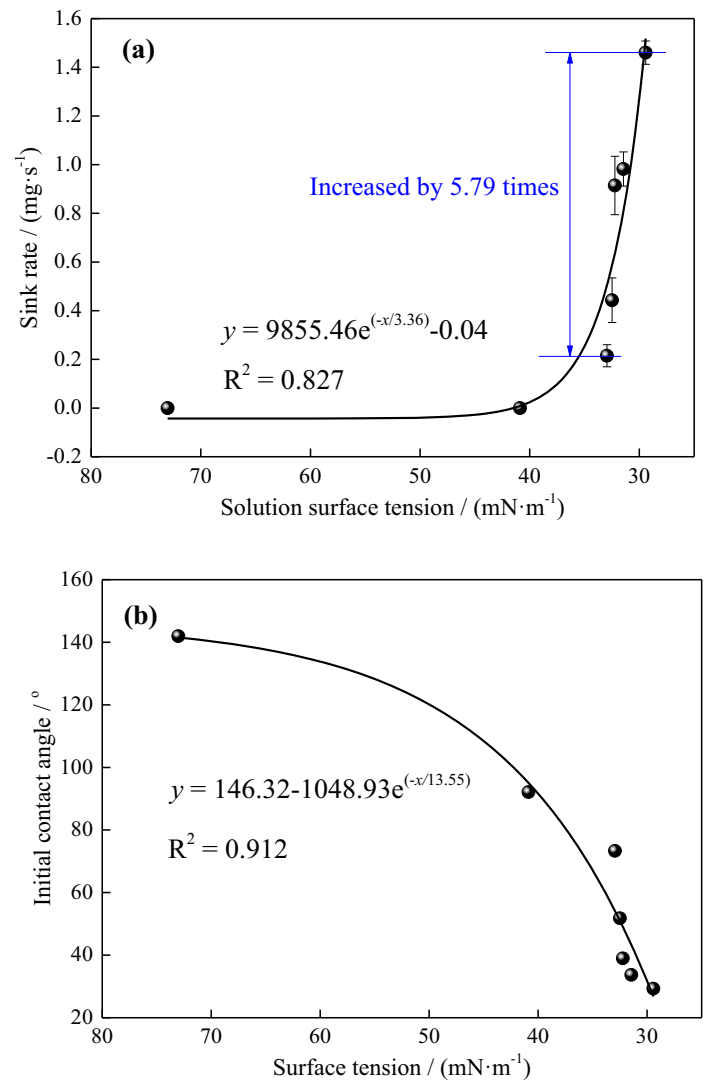

Fig. 11 Relationship between the solution surface tension and the $S R$ (a) and ICA (b) of coal dust

best concentration of dust suppressants. In addition, decreasing the surface tension of the solution can improve its wettability for coal dust, but their relationship is nonlinear.

\subsubsection{Sink rate}

The sink test is one of the most accurate laboratory tests to evaluate the wettability of coal dust (Chen et al. 2019). As shown in Fig. 10, the coal dust does not settle in clean water or a $0.1 \mathrm{wt} \% \mathrm{NCZ}$ solution, but it begins to sink in $\mathrm{NCZ}$ solutions with concentrations above $0.5 \%$. As seen, the settling rate gradually increased with the NCZ concentration. The sink rate was improved by $45.0 \%$ when the $\mathrm{NCZ}$ concentration varied from $10.0 \mathrm{wt} \%$ to $20.0 \mathrm{wt} \%$, which differs greatly from the changes in the surface tension that only reduced by $1.0 \mathrm{mN} / \mathrm{m}$ over the same concentration range. This demonstrates that the solution surface tension changes a little after the $\mathrm{CMC}$, but its wettability of coal dust does not reach its maximum there. Thus, the wettability of the solution for coal dust can still be improved considerably by increasing the NCZ concentration beyond its CMC. 


\subsection{Effect of solution surface tension on the wettability of coal dust}

Numerous studies have shown that the wettability of a suppressant solution to coal dust increases with a lower surface tension (Xu et al. 2018; Zhou G et al. 2018; Zhou L et al. 2018; Zhou Q et al. 2018; Shi et al. 2019; Xi et al. 2019). However, a quantitative analysis of this phenomenon has not yet been thoroughly conducted, which is significant to guide field applications of chemical dust suppressants and improve their efficiencies. The relationship between the solution surface tension and the wettability of coal dust (including $S R$ and ICA) is fit using the least squares method based on the obtained test data. Figure 11a illustrates the relationship between the solution surface tension and the $S R$ of coal dust. An exponential function is obtained with a goodness of fit $\left(R^{2}\right)$ of 0.827 . This indicates that the $S R$ of coal dust exponentially increases with decreases in the solution surface tension. The $S R$ of coal dust increased by 5.79 times when the solution surface tension decreased from 32.95 to $29.42 \mathrm{mN} /$ m.

As shown in Fig. 11b, a negative exponential function is established between the solution surface tension and the ICA of coal dust. Without considering cost, a smaller surface tension for the solution gives a better wetting performance on coal dust. This indicates that the wettability of solutions to coal dust is exponentially related (i.e., non-linear) with its surface tension. Therefore, this is the most effective method to improve the dust suppression efficiency through continual reductions in the solution surface tension.

\section{Conclusions}

A novel composite dust suppressant $\mathrm{NCZ}$ was prepared that utilized $\mathrm{CaCl}_{2}, \mathrm{MgCl}_{2}$, and nonionic surfactants for hydrophobic coal dust. Its water-retaining properties and wettability of coal dust were comprehensively tested in a laboratory environment. The following conclusions are drawn:

(1) The critical micelle concentration (CMC) and the corresponding surface tension of the NCZ solution were $0.5 \mathrm{wt} \%$ and $32.95 \mathrm{mN} / \mathrm{m}$, respectively. The surface tension of the NCZ solution was reduced to $29.42 \mathrm{mN} / \mathrm{m}$ when its concentration increased to $20.0 \mathrm{wt} \%$.

(2) The water-absorbing process of the NCZ powder involved three stages: physical adsorption stage (0-130 min), chemical adsorption stage (130-400 $\mathrm{min})$, and equilibrium adsorption and evaporation stage ( $>400 \mathrm{~min}$ ). Additionally, the $E R$ of the $20.0 \mathrm{wt} \% \mathrm{NCZ}$ solution was reduced by $11.7 \%$ relative to clean water.

(3) The NCZ solution had a remarkable enhancement effect on the wettability of coal dust. The ICA and $S R$ for clean water and the NCZ solution (20.0 wt $\%$ ) were $141.9^{\circ}$ and $0 \mathrm{mg} / \mathrm{s}$, and $29.3^{\circ}$ and $1.46 \mathrm{mg} / \mathrm{s}$, respectively.

(4) The solution surface tension was exponentially related to the $S R$ and ICA. Thus, the wettability of coal dust can be rapidly improved by continually decreasing the solution surface tension when it is below $32 \mathrm{mN} / \mathrm{m}$. Without considering costs, a smaller solution surface tension gives a better wetting performance on coal dust.

Finally, the limitations of this study are that the mechanism of the exponential relationship between the surface tension and SR and ICA has not been analyzed or developed theoretically or experimentally, which we intend to achieve in future work.

Acknowledgements We much appreciate the National Nature Science Foundation of China (Nos. 51874015 and 51574017) and the National Key Research and Development Program of China (No. 2017YFC0805204).

Authors' Contributions Jianguo Liu conceived and designed the investigation, collected data, performed the analysis, and wrote the paper. Shu Wang helped for designing the experiments and co-wrote the paper. Longzhe Jin was the academic supervisor who conceived the study and provided financial support. Tianyang Wang, Zihao Zhou, and Jingge $\mathrm{Xu}$ helped for the experiments.

\section{Compliance with ethical standards}

Conflict of interest The author declare that they have no conflict of interest.

Open Access This article is licensed under a Creative Commons Attribution 4.0 International License, which permits use, sharing, adaptation, distribution and reproduction in any medium or format, as long as you give appropriate credit to the original author(s) and the source, provide a link to the Creative Commons licence, and indicate if changes were made. The images or other third party material in this article are included in the article's Creative Commons licence, unless indicated otherwise in a credit line to the material. If material is not included in the article's Creative Commons licence and your intended use is not permitted by statutory regulation or exceeds the permitted use, you will need to obtain permission directly from the copyright holder. To view a copy of this licence, visit http://creativecommons. org/licenses/by/4.0/.

\section{References}

Bao Q, Nie W, Liu CQ, Zhang HH, Wang HK, Jin H, Yan JY, Liu Q (2020) The preparation of a novel hydrogel based on crosslinked polymers for suppressing coal dusts. J Clean Prod 249:119343 
Berry JD, Neeson MJ, Dagastine RR, Chan DYC, Tabor RF (2015) Measurement of surface and interfacial tension using pendant drop tensiometry. J Colloid Interface Sci 454:226-237

Chen YP, Xu G, Huang JX, Eksteen J, Liu XF, Zhao ZD (2019) Characterization of coal particles wettability in surfactant solution by using four laboratory static tests. Colloids Surf A 567:304-312

Hall NB, Blackley DJ, Halldin CN, Laney AS (2019) Continued increase in prevalence of r-type opacities among underground coal miners in the USA. Occup Environ Med 76:479-481

Jin H, Nie W, Zhang HH, Liu YH, Bao Q, Wang HK, Huang DM (2019) Preparation and characterization of a novel environmentally friendly coal dust suppressant. J Appl Polym Sci 136:47354

Jin LZ, Jiang TG, Jin AY (1999) Characteristics of water absorption and the loss of dust-sticking agent. J Miner Metall Mater 6:1-3

Kollipara VK, Chugh YP, Mondal K (2014) Physical, mineralogical and wetting characteristics of dusts from Interior Basin coal mines. Int J Coal Geol 127:75-87

Li QZ, Lin BQ, Zhao S, Dai HM (2013) Surface physical properties and its effects on the wetting behaviors of respirable coal mine dust. Powder Technol 233:137-145

Liu JG, Jin LZ, Wang JY, Ou SN, Wang TY (2019a) Co-influencing mechanisms of physicochemical properties of blasting dust in iron mines on its wettability. Int J Miner Metall Mater 26:1080-1091

Liu JG, Jin LZ, Wang JY, Ou SN, Guo JZ, Wang TY (2019b) Micromorphology and physicochemical properties of hydrophobic blasting dust in iron mines. Int $\mathrm{J}$ Miner Metall Mater 26:665-672

Meng JQ, Yin FF, Li SC, Zhong RQ, Sheng ZY, Nie BS (2019) Effect of different concentrations of surfactant on the wettability of coal by molecular dynamics simulation. Int J Mining Sci and Technol 29:577-584

Ni GH, Sun Q, Xun M, Wang H, Xu YH, Chen WM, Wang G (2019) Effect of NaCl-SDS compound solution on the wettability and functional groups of coal. Fuel 257:116077

Notsu H, Kubo W, Shitanda I, Tatsuma T (2005) Super-hydrophobic/super-hydrophilic patterning of gold surfaces by photocatalytic lithography. J Mater Chem 15:1523-1527

Shi GQ, Han C, Wang YM, Wang HT (2019) Experimental study on synergistic wetting of a coal dust with dust suppressant compounded with noncationic surfactants and its mechanism analysis. Powder Technol 356:1077-1086

Wang HT, Zhang L, Wang DM, He XX (2017) Experimental investigation on the wettability of respirable coal dust based on infrared spectroscopy and contact angle analysis. Adv Powder Technol 28:3130-3139

Wang PF, Tan XH, Zhang LY, Li YJ, Liu RH (2019) Influence of particle diameter on the wettability of coal dust and the dust suppression efficiency via spraying. Process Saf Environ 132:189-199

Xi X, Jiang SG, Zhang WQ, Wang K, Shao H, Wu ZY (2019) An experimental study on the effect of ionic liquids on the structure and wetting characteristics of coal. Fuel 244:176-183

Xi ZL, Feng ZY, Li A (2017) Synergistic coal dust control using aqueous solutions of thermoplastic powder and anionic surfactant. Colloids Surf A 520:864-871

$\mathrm{Xu} \mathrm{CH}$, Wang DM, Wang HT, Xin HH, Ma LY, Zhu XL, Zhang Y, Wang QG (2017) Effects of chemical properties of coal dust on its wettability. Powder Technol 318:33-39

Xu G, Chen YP, Eksteen J, Xu JL (2018) Surfactant-aided coal dust suppression: a review of evaluation methods and influencing factors. Sci Total Environ 639:1060-1076

Zhang HH, Nie W, Wang HK, Qiu B, Hu J, Liu YH (2018) Preparation and experimental dust suppression performance characterization of a novel guar gum-modification-based environmentally-friendly degradable dust suppressant. Powder Technol 339:314-325

Zhou G, Ding JF, Ma YL, Li SL, Zhang MG (2020) Synthesis and performance characterization of a novel wetting cementing agent for dust control during conveyor transport in coal mines. Powder Technol 360:165-176

Zhou G, Ma YL, Fan T, Gang W (2018a) Preparation and characteristics of a multifunctional dust suppressant with agglomeration and wettability performance used in coal mine. Chem Eng Res Des 132:729-742

Zhou L, Yang SY, Hu B, Yuan ZL, Wu H, Yang LJ (2018b) Evaluating of the performance of a composite wetting dust suppressant on lignite dust. Powder Technol 339:882-893

Zhou Q, Qin BT, Wang J, Wang HT, Wang F (2018c) Effects of preparation parameters on the wetting features of surfactantmagnetized water for dust control in Luwa mine, China. Powder Technol 326:7-15 